\title{
Exploring interstellar titanium and deuterium abundances and other correlations ${ }^{\star}$
}

\author{
R. Lallement ${ }^{1}$, G. Hébrard ${ }^{2}$, and B. Y. Welsh ${ }^{3}$ \\ 1 Service d'Aéronomie, UMR7620 CNRS, Université Versailles Saint-Quentin, BP 3, 91371 Verrières-le-Buisson, France \\ e-mail: rosine.lallement@aerov.jussieu.fr \\ 2 Institut d'Astrophysique de Paris, UMR7095 CNRS, Université Pierre \& Marie Curie, 98bis boulevard Arago, 75014 Paris, France \\ e-mail: hebrard@iap.fr \\ 3 Space Sciences Laboratory, University of California, 7 Gauss Way, Berkeley, CA 94720, USA \\ e-mail: bwelsh@ssl.berkeley.edu
}

Received 9 October 2007 / Accepted 3 January 2008

\section{ABSTRACT}

\begin{abstract}
Aims. The origin of the observed variability of the gas-phase $\mathrm{D} / \mathrm{H}$ ratio in the local interstellar medium is still debated, and in particular the role of deuterium depletion onto dust grains. Here we extend the study of the relationship between deuterium and titanium, a refractory species and tracer of elemental depletion, and explore other relationships.

Methods. We have acquired high resolution spectra for nine early-type stars using the VLT/UVES spectrograph, and detected the absorption lines of interstellar TiII. Using a weighted orthogonal distance regression (ODR) code and a special method to treat non symmetric errors, we compare the TiII columns with the corresponding HI, DI and also OI columns. In parallel we perform the same comparisons for available FeII data.

Results. We find a significant correlation between TiII/HI and D/H in our data set, and, when combined with published results, we confirm and better constrain the previously established trends and extend the trends to low HI columns.

We exclude uncertainties in $\mathrm{HI}$ and $\mathrm{OI}$ columns as the main contributor to the derived metals-deuterium correlations by showing that the TiII/HI ratio is positively correlated with DI/OI. We find a similar correlation between FeII/HI and DI/OI.

The TiII gradients are similar or slightly smaller than for FeII, while one would expect larger variations on the basis of the higher condensation temperature of titanium. However we argue that ionisation effects introduce biases that affect iron and not titanium and may explain the gradient similarity.

We find a less significant negative correlation between the TiII/DI ratio and the hydrogen column, possibly a sign of different evaporation of $\mathrm{D}$ and metals according to the cloud properties. More Till absorption data along very low $\mathrm{H}$ column lines-of-sight would be useful to improve the correlation statistics.
\end{abstract}

Key words. ISM: abundances - ISM: evolution - Galaxy: evolution - Galaxy: solar neighborhood

\section{Introduction}

The measurement of the present-day ratio of deuterium (D) to hydrogen $(\mathrm{H})$ in the Galaxy places important constraints on its chemical evolution and has been the goal of intense work, from the Copernicus era (Rogerson \& York 1973) to HST (e.g. Linsky 1998), IMAPS (Jenkins et al. 1999; Sonneborn et al. 2000) and FUSE (e.g. Moos et al. 2002). Measurements inside the Local Bubble (LB), the volume of tenuous gas surrounding the Sun (e.g. Snowden et al. 1998; Welsh et al. 1999; Lallement et al. 2003) appear to be consistent with a single value for D/H (Moos et al. 2002; Hébrard \& Moos 2003; Oliveira et al. 2003), but other measurements (e.g. Jenkins et al. 1999; Sonneborn et al. 2000; Hoopes et al. 2003; Friedman et al. 2006; Hébrard et al. 2005; Oliveira \& Hébrard 2006) suggest variations of the interstellar $\mathrm{D} / \mathrm{H}$ ratio at larger distances, i.e. beyond $\simeq 150-200$ parsecs. Quantitatively, within and at the periphery of the $\mathrm{LB}\left(\log N(\mathrm{HI}) \leq 19.3 \mathrm{~cm}^{-2}\right)$, the $\mathrm{D} / \mathrm{H}$ ratio is of the order of $15 \mathrm{ppm}$ (see Fig. 1 of Linsky et al. 2006), while lines-of-sight measurements with higher $N(\mathrm{HI})$ show a strong variability, with maximum values of $\simeq 23 \mathrm{ppm}$. For a number of lines-of-sight (LOS) with $\log N(\mathrm{HI}) \leq 20.5 \mathrm{~cm}^{-2}, \mathrm{D} / \mathrm{H}$ is significantly lower, $\simeq 7 \mathrm{ppm}$.
According to Draine (2004) and Linsky et al. (2006) (see also Linsky 2007), the wide range in the gas-phase $\mathrm{D} / \mathrm{H}$ ratios is due to different amounts of deuterium depletion onto interstellar grains. Theoretical support for this interpretation is the preferential adsorption of deuterium onto interstellar grains, in particular PAHs (Jura 1982; Draine 2004). Observational support comes from the correlations between $\mathrm{D} / \mathrm{H}$ and both $\mathrm{Fe}$ and Si depletions, as well as the correlations with the rotational temperatures of $\mathrm{H}_{2}$ for lines-of-sight possessing molecular gas (Linsky et al. 2006). The $\mathrm{D} / \mathrm{H}$ trends quoted above may be explained by the depletion hypothesis if one hypothesizes a recent heating of the LB and especially recent shocks in star-forming regions at its periphery. This interpretation has important consequences and implies that the total $\mathrm{D} / \mathrm{H}$ ratio including deuterium in the gas and in the dust is at least 23 parts per million of hydrogen, which poses a challenge for models of galactic chemical evolution.

On the other hand, Oliveira \& Hébrard (2006) show that high $\mathrm{D} / \mathrm{H}$ values are also found along distant lines-of-sight with associated high interstellar columns. At high $N(\mathrm{HI})$ the selection bias against high $\mathrm{D} / \mathrm{H}$ values (due to dominance of dense, cold, and thus strongly depleted regions) should not lead to any variability (Oliveira et al. 2006). Moreover, D/O measurements do not fully support this depletion scenario, since the DI/OI ratio is found to 
Table 1. Summary of observations and titanium results.

\begin{tabular}{cccclllll}
\hline \hline Target & III & bII & $d(\mathrm{pc})$ & type & Obs-Date & Exp-time $(\mathrm{s})$ & $S N$ & $\log N(\mathrm{TiII})$ \\
\hline$\alpha$ Vir & 316.1 & 50.8 & $80 \pm 6$ & B1III-IV & $22-05-06$ & $50 \times .35$ & 380 & $10.49 \pm 0.13$ \\
$\beta$ Cen & 311.8 & 1.2 & $161 \pm 15$ & B1III & $15-04-06$ & $50 \times 0.2$ & 340 & $10.99 \pm 0.04$ \\
$\alpha$ Cru & 300.1 & -0.36 & $98 \pm 6$ & B & $10-04-06$ & $50 \times 0.15$ & 290 & $11.12 \pm 0.035$ \\
$\gamma^{2}$ Vel & 262.8 & -7.7 & $368(+38-13)^{1}$ & WR+O & $09-04-06$ & $50 \times 0.6$ & 320 & $11.11 \pm 0.035$ \\
$\mu$ Col & 237.2 & -27.1 & $396 \pm 100$ & O9.5 & $01-04-06$ & $40 \times 8$ & 310 & $11.50 \pm 0.02$ \\
$\zeta$ Pup & 256.0 & -4.7 & $429 \pm 94$ & O5I & $29-03-06$ & $45 \times 0.7$ & $250^{*}$ & $11.16 \pm 0.06$ \\
$\theta$ Car & 289.6 & -4.9 & $135 \pm 9$ & B0V & $10-04-06$ & $40 \times 0.9$ & $320^{*}$ & $11.31 \pm 0.023$ \\
JL9 & 322.6 & -27.0 & $590 \pm 160$ & sdO & $19-05-06$ & $2 \times 2700$ & 280 & $11.88 \pm 0.10$ \\
LS 1274 & 277.0 & -5.3 & $580 \pm 100$ & sdB & $10-04-06$ & $2 \times 2100$ & 110 & $11.68 \pm 0.04$ \\
\hline
\end{tabular}

${ }_{1}^{1}$ Distance recently revised by Millour et al. (2007). *: $S / N$ after fringe correction.

be more constant than DI/HI (Hébrard et al. 2005). Interestingly the $\mathrm{LB} \mathrm{D} / \mathrm{H}$ ratio derived from the $\mathrm{D}$ and $\mathrm{O}$ measurements is only of the order of 13 ppm (Hébrard \& Moos 2003). These authors suggest that the uncertainties in the measurement of $\mathrm{HI}$ in the data play an important role in the subsequently derived relationships. Two quotients with the same denominator remain naturally correlated if there are measurement errors on this denominator. FeII/HI vs. DI/HI or FeII/OI vs. DI/OI relationships could result from this effect.

Infall of external unprocessed (or less processed) gas also creates $\mathrm{D} / \mathrm{H}$ variability, and indeed a continuous infall is a general property in the Galaxy (Romano et al. 2006). Support for the existence of a significant infall into the Sun's neighborhood comes from $\mathrm{D}, \mathrm{O}, \mathrm{N}$, and ${ }^{3} \mathrm{He}$ abundance values found in the local interstellar cloud that surrounds the Sun. Geiss et al. (2002) interpret these abundances as being due to the local mixture of processed galactic gas and very weakly processed (Magellanic type) gas of intergalactic origin having fallen onto the disk. Such an infall could have occurred recently, leading to a present-day incomplete mixing. On the other hand, the wide $\mathrm{D} / \mathrm{H}$ range implies a non realistic infall strength and a very weak mixing after the infall, as argued by Linsky et al. (2006).

It is therefore mandatory to investigate in more detail the depletion mechanisms and the associated deuterium behaviour. Prochaska et al. (2005) made an important step by showing a positive correlation between the abundance of titanium, a refractory species, and that of $\mathrm{D} / \mathrm{H}$. Here we present results we have obtained in the southern hemisphere with the Ultraviolet and Visual Echelle Spectrograph (UVES) at the ESO Very Large Telescope (VLT), and we add nine new TiII measurements towards $\mathrm{D} / \mathrm{H}$ targets. In parallel to our observations and analysis, Ellison et al. (2007) also gathered and analysed new VLT-UVES and Keck Observatory HIRES data. We have combined our results with their results and those from Prochaska et al. For targets in common, we have compared the two results. We have extended the correlative study, in particular to lower columndensities. We have used sophisticated methods to treat asymmetric errors in both correlated quantities.

Titanium is a particularly well suited element for the study of a potential correlation between depletion and deuterium abundance. It has a high depletion which is linked to its high condensation temperature. TiII is optically accessible in the near ultraviolet via the $\lambda \lambda 3229.199,3242.994,3383.768 \AA$ absorption lines (wavelengths from Morton 1991), and interstellar titanium has been observed in absorption since the 1970's (e.g. Wallerstein \& Goldsmith 1974; Stokes 1978; Hobbs 1984). Moreover, as already argued by these authors, Till is the dominant ionization state in HI regions due to the nearly exact coincidence of the ionization potentials of TiII and HI. Also, TiII is coupled to HI via charge-exchange reactions (Field \& Steigman 1971). The observed close $N(\mathrm{TiII})$ vs. $N(\mathrm{HI})$ relationship, in contrast with the more dispersed $N(\mathrm{NaI}) / N(\mathrm{HI})$ or $N(\mathrm{CaII}) / N(\mathrm{HI})$ relations (see e.g. Fig. 7 of Welsh et al. 1997), results from those physical properties.

The detection of the interstellar Till lines requires high spectral resolution $(R>50000)$ and appropriate optical systems and detectors adapted to the near ultraviolet wavelength range. Prochaska et al. (2005) used HIRES at the Keck Observatory and observed absorption towards 7 northern hemisphere stars. Due to this limited number of targets, it is important to extend their study to other $\mathrm{D} / \mathrm{H}$ targets. Moreover, the observed trend is strongly dependent on the measurement towards Feige 110, and indeed Hébrard et al. (2005) have shown that the column of HI is probably significantly underestimated for this star. They also quote the contradicting case of $\gamma^{2} \mathrm{Vel}$, a line-of-sight with high $\mathrm{D} / \mathrm{H}$ and low titanium abundance. Finally, while they covered a range of $\log N(\mathrm{HI})$ from 19.85 and $21.05 \mathrm{~cm}^{-2}$, it is important to extend the study to lines-of-sight with lower HI columns, because there is a strong $\mathrm{D} / \mathrm{H}$ variability for $\log N(\mathrm{H}) \leq 20 \mathrm{~cm}^{-2}$ and no titanium column density data are currently available within this range.

Section 2 describes the observations, data analysis and Till column determination. In Sect. 3 we perform linear comparison fits between TiII and other species, using our data in combination with other published data. We perform corresponding correlative studies for available FeII data. Section 4 discusses the results.

\section{Observations and absorption line analysis}

The spectroscopic data were obtained with the ESO VLT-UT2 telescope and the UVES spectrograph (Dekker et al. 2000) under the observing program 077.C-0547A. We used the standard dichroic 346-580 $\mathrm{nm}$ setting (DIC1) and the image slicer 1 which corresponds to $R \simeq 60000$ at $3400 \AA$. This setup included the $\lambda \lambda 3229,3242,3384 \AA$ TiII lines with the blue arm. The spectra were recorded in service mode.

The targets were seven bright early-type stars and two hot subdwarfs. They were selected from the list of D/H targets stars recently compiled by Linsky et al. (2006). Multiple short exposures were obtained for each of the bright stars and two long exposures for the subdwarfs. Details of the observations are listed in Table 1. The individual stellar spectra, processed through the UVES pipeline, have been co-added.

The spectral analysis and the correlative studies were performed in the frame of the IGOR/WaveMetrics software, using both built-in and specially developed routines. In the present 

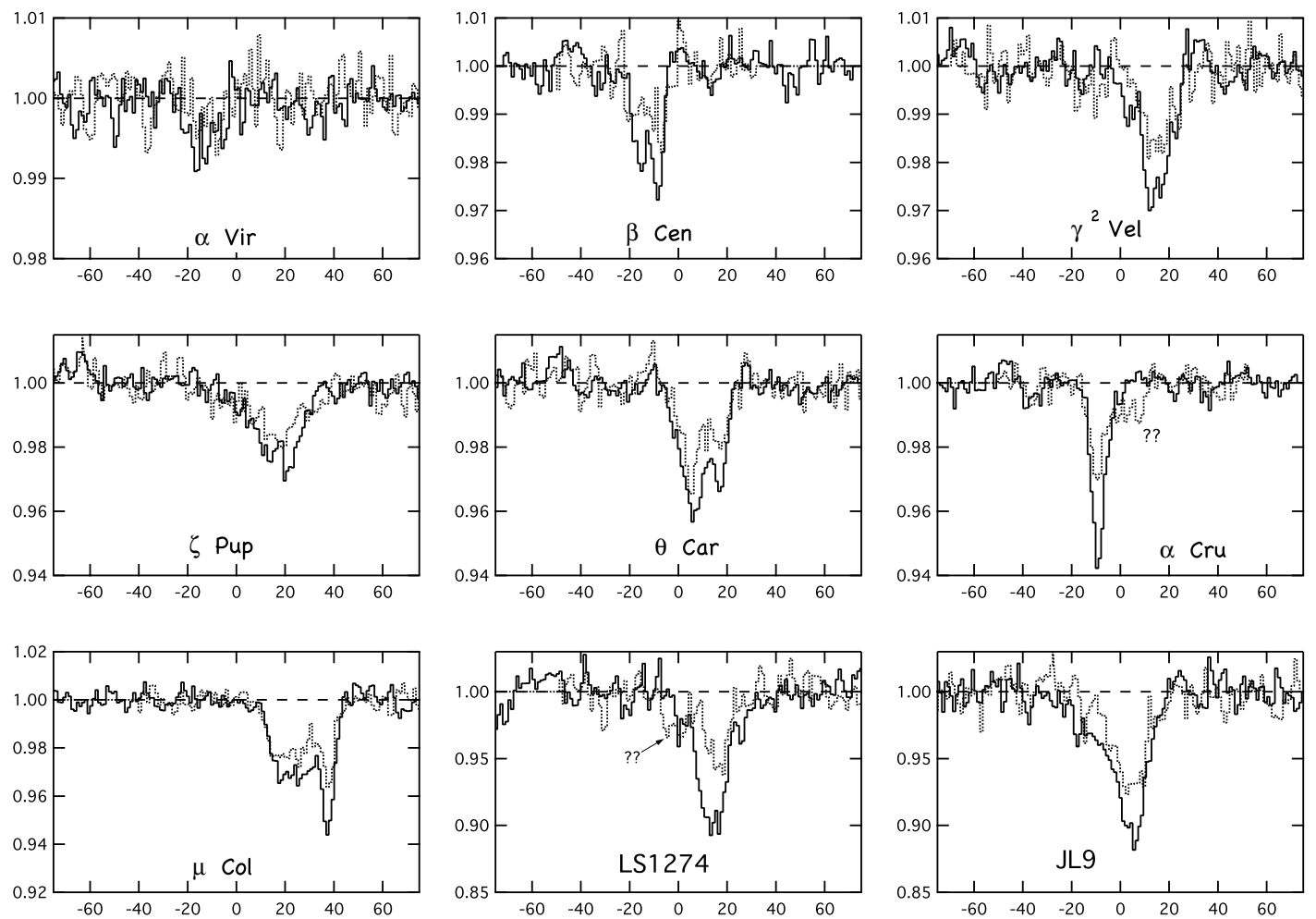

Fig. 1. Normalised spectra around the TiII $3384 \AA$ (solid lines) and $3242 \AA$ (dashed lines) transitions for our target stars. The abscissa is the heliocentric velocity in $\mathrm{km} \mathrm{s}^{-1}$.

work we are interested in the total Till column-density values (a cloud-by-cloud analysis is beyond its scope), and since we are in the optically thin linear regime measurement of the total equivalent width is sufficient. For this purpose, stellar continua around the strongest transitions $\lambda \lambda$ 3383.768, $3241.994 \AA$ were fit with a multi-order polynomial. The noise level was automatically calculated during the stellar continuum fit and assumed to prevail at the absorption line locations. The resulting signal to noise ratios around the $3384 \AA$ transition are given in Table 1. The normalised spectra corresponding to the two strongest transitions are displayed in Fig. 1. Note that these targets stars correspond to a two orders of magnitude variation of the HI column-density.

After division by the fitted continuum, equivalent widths are simply deduced from the normalised spectra. Errors on the total TiII equivalent width are estimated on the basis of the noise level and the number of pixels defining the absorption.

Two of the target spectra ( $\theta$ Car, $\zeta$ Pup) are affected by some instrumental interference fringes. For these stars the stellar continua were fit with the sum of a multi-order polynomial and a sinusoidal function with an adjustable period, phase and amplitude. The correction is illustrated in Fig. 2 for $\theta$ Car which corresponds to the maximum fringe amplitude. The difference in derived equivalent widths (or column densities) with and without the fringe correction is generally less than the error resulting from the noise.

The column-densities of TiII are deduced here from the strongest $3384 \AA$ transition. On the basis of experimental data Pickering et al. (2001) have revised the oscillator strength of this transition at $f=0.358$, some 5 percent higher than the Morton (1991) value $f=0.3401$. In order to be consistent with the previous analyses of Prochaska et al. and Ellison et al. we have used this revised value. Note that the results of this work and previous ones on the existence and quality of correlations remain
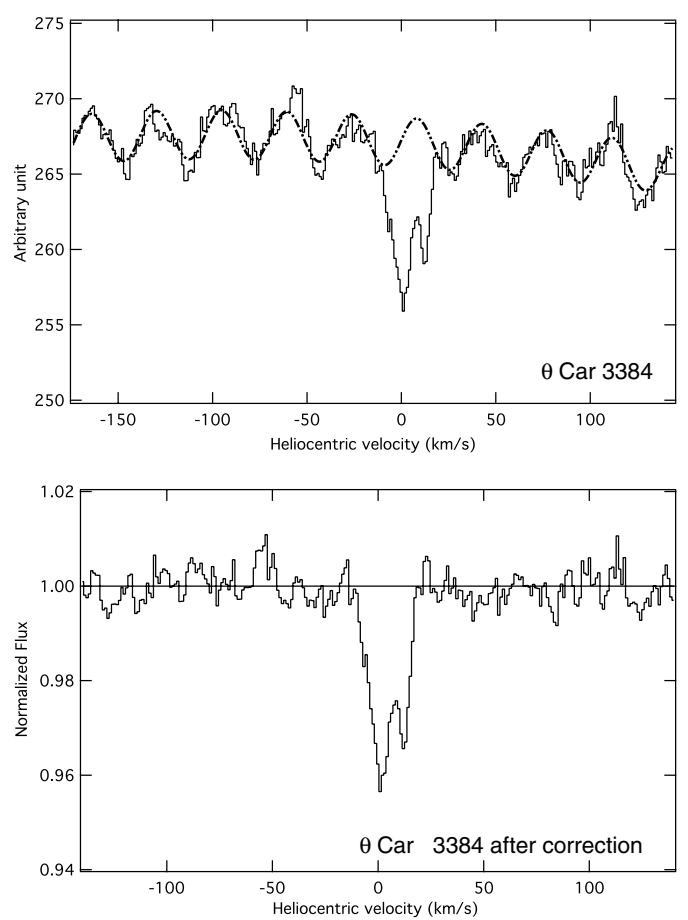

Fig. 2. Illustration of fringe correction to the data: the extreme case of $\theta$ Car.

unchanged if this oscillator strength is revised again. Only resulting gradients would have to be scaled according to the changes.

For the $\lambda \lambda 3242 \AA$ transition we first used the Morton (1991) atomic data, then the revised values quoted by Prochaska et al. (2005). Their atomic data obtained from Morton (2003, private communication) are significantly different from the older ones, 

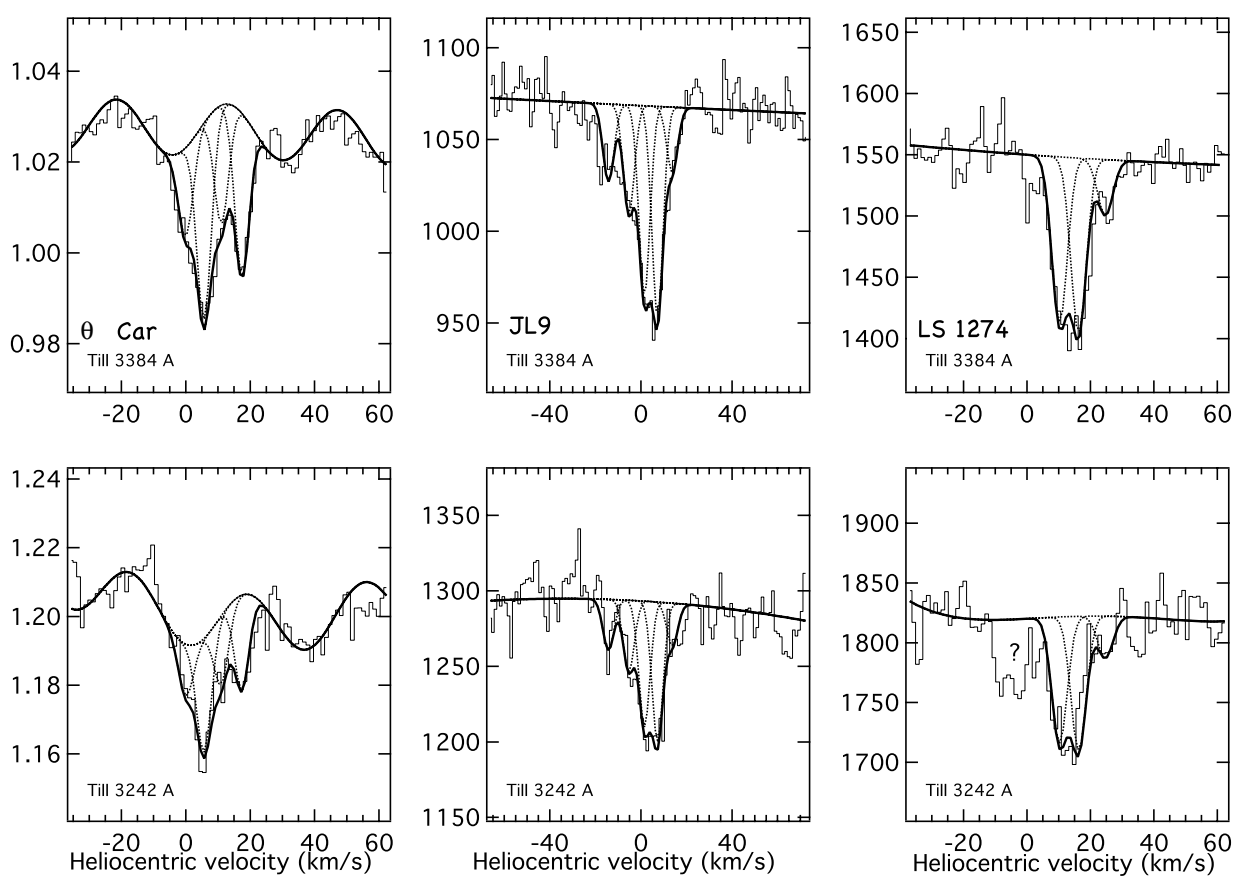

Fig. 3. 3394-3242 A simultaneous line-fitting used as a consistency checked: $\theta$ Car, JL9, and LS1274. The blue-shifted absorption at $3384 \AA$ in LS1274 has no counterpart at $3242 \AA$ and is very likely stellar (see text).
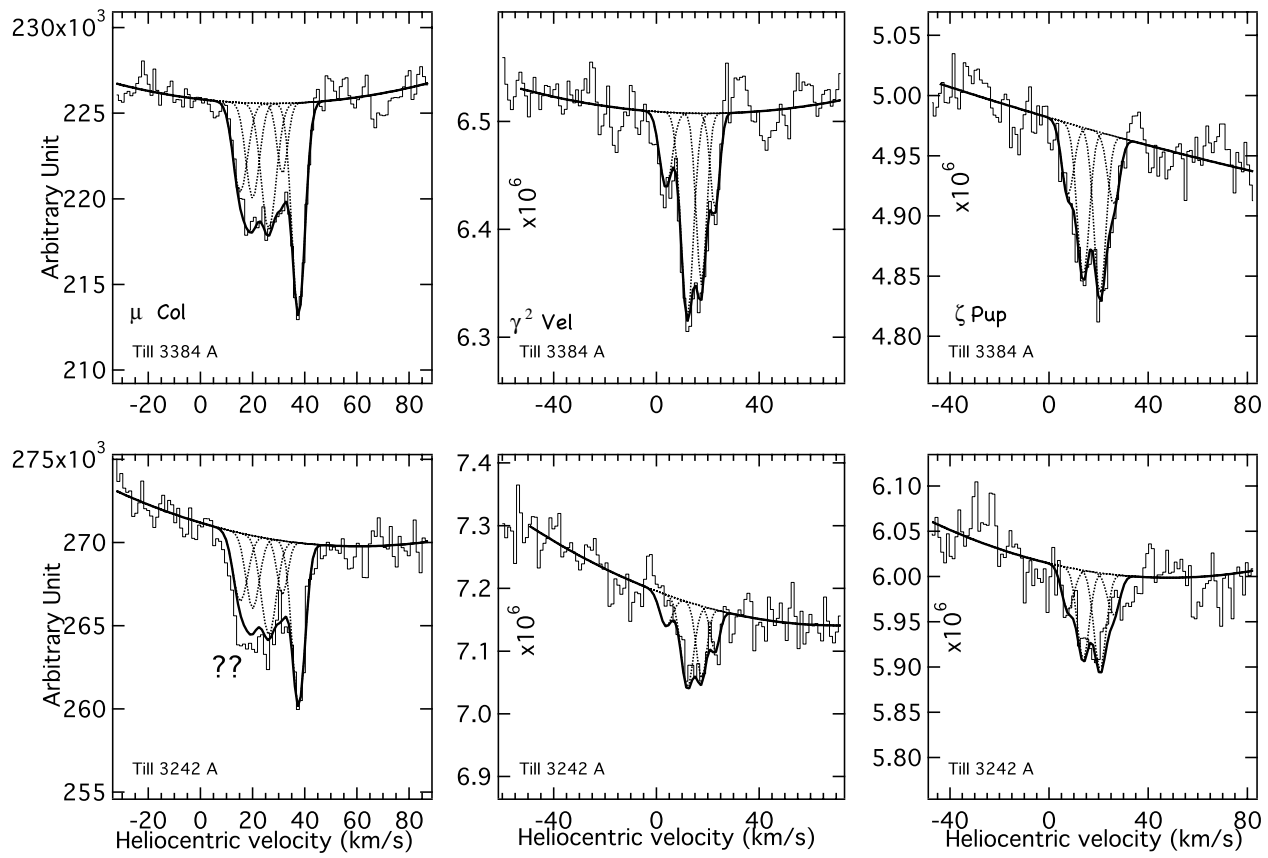

Fig. 4. Same as Fig. 2: $\gamma^{2}$ Vel, $\zeta$ Pup, and $\mu$ Col. The broad feature in $\mu$ Col cannot be fitted satisfyingly (see text).

with the oscillator strength increased from $f=0.183$ to 0.232 . We could check from the simultaneous fits of the two lines that those revised values are in better agreement with data than the Morton (1991) values and we show only the second results.

We have also performed line-fitting, using the program described in Sfeir et al. (1999), i.e., the residual intensity absorption profiles were fit to combinations of Voigt profiles, convolved by the UVES instrumental function. We have limited the temperature to the 100-15000 K range, which corresponds to realistic values in the local ISM, and we have simply chosen the minimum number of clouds allowing a good visual adjustment to the data. In parallel we performed a profile fitting analysis with the
Owens.f software of Lemoine et al. (2002) for one of the targets and the results are consistent. The cloud-by-cloud structure in those line-fitting results should not be considered as the best solutions, since they should also be compared in future work with ithe structure derived from other elements. However, they provide a consistency check. Simultaneous fitting of the two transitions are shown in Figs. 3 and 4. We have not included $\alpha$ Vir, $\beta \mathrm{Cen}$ and $\alpha \mathrm{Cru}$, for which the column is small and the detection of the weakest line is of insufficient quality and does not add to the $3384 \AA$ detection.

Although we do not specifically use the column densities or the cloud-by-cloud structure resulting from this exercise, fitting 
the two 3384 and $3242 \AA$ lines, as said above, has provided a consistency check: Lines other than TiII and (or) non-random noise features are revealed by this procedure.

While performing this exercise we noticed the following departures from the model absorptions. For LS1274 the $\lambda \lambda 3241.994 \AA$ region is affected by an additional absorption that has no counterpart at $3384 \AA$ not at $3229 \AA$ (the absorption is strong enough for this star to be detectable at this wavelength). Looking at the entire spectrum, we found that at variance with the other targets and especially the second subdwarf JL9, the spectrum is characterized by a very large number of narrow features. We could not determine whether these narrow lines have a circumstellar or stellar origin (LS1274 is cooler than JL9) origin, and their study is beyond the scope of this paper. For this star we have calculated the column-density without including this additional absorption that is not seen in the stronger transition and is indicated in Fig. 1. A second, although smaller, anomaly is found for $\alpha \mathrm{Cru}$, for which there is an absorption feature seen at $3342 \AA$ and not for the strongest transition. The noise level however is such that a non-random noise feature may be responsible. After examination of the two transitions and of these line fit results we have accordingly increased the error bars on the equivalent widths and resulting columns to take into account such non random noise features.

We also have searched for a possible contamination by a stellar line by shifting each spectrum to the stellar reference frame and overplotting the resulting shifted spectra. No particular absorption at a given wavelength is revealed by such a method. The two subdwarfs however are excluded because stellar radial velocities for those targets are uncertain.

For all targets except $\mu \mathrm{Col}$, the equivalent width ratios are compatible with the theoretical value of $\simeq 1.65$ deduced from the revised atomic data, when taking into account noise features and the LS1274 blend, and it is possible to obtain a reasonable simultaneous fit to the two transitions. Although in the case of $\mu \mathrm{Col}$ the spectrum is of excellent quality, and the two transitions clearly show the same structure, we could not fit them simultaneously in a satisfying way (see Fig. 3). More specifically, the narrow line in the red is correctly fitted, but the broad feature in the blue is not, and the probability of this being due to random noise is less than $5 \%$ ( $2 \sigma$ equivalent width deficit). We suspect a non-interstellar origin for this broad absorption, and will discuss the peculiarity of this star later.

We have also verified that for all our targets neutral titanium (TiI) is negligible with respect to TiII (see Sect. 1). For that purpose we checked for the presence of $\lambda \lambda 3342,3636 \AA$ TiI absorption lines within the velocity interval deduced from the observed TiII lines. We could not detect any line absorption. Since oscillator strengths for those TiI transitions are similar to the TiII ones, this implies that the contribution from TiI is negligible.

\section{Comparisons between Till, DI, HI, OI, Fell}

\subsection{Methods and selected targets}

In the following sections, we have combined our titanium data (9 targets covering $\log N(\mathrm{HI})$ from 19.0 to 20.98) with those of Prochaska et al. (7 stars with $\log N(\mathrm{HI})$ from 19.85 to 21.05). For all abundance correlations we have adopted the $\mathrm{D} / \mathrm{H}$ ratio, $\log N(\mathrm{HI}), N(\mathrm{DI})$ and iron depletion, $\mathrm{D}(\mathrm{Fe})$, compiled in Table 1 of Linsky et al. (2006). In addition, we have used the DI/OI ratios compiled in Table 12 of Oliveira et al. (2006). All the data and errors used in this article are listed in Table 2. For all correlations we have made use of the maximum amount of published data.
Because OI and FeII measurements are not available for exactly the same targets, the correlation fits use different target lists.

After completion of this work, Ellison et al. (2007) made available results from new Keck and UVES TiII measurements. Some of their targets are already included in our new list. For those targets we have used our spectral results, resulting columns and errors. For those targets in common we also checked for consistency of the two measurements and found that our column density intervals overlap (see the caption of Table 2). There are 4 additional stars in Ellison et al. that were not included in our study. We have added these 4 targets in Table 2, and we have performed the same correlation fits with and without those four targets based on the correlation analysis method discussed below. Four of our measurements are not part of the Ellison et al. study, the low column target $\alpha$ Vir, the two faint subdwarfs, and $\mu \mathrm{Col}$, for which Ellison et al. had an upper limit only.

We have also compared our column density results with previous measurements and analyses. We have disagreements with the results of Welsh et al. (1997).

While for $\mu \mathrm{Col}$ we differ by $10 \%$ only, in the case of $\zeta$ Pup our column $\log ($ TiII $)=11.18 \pm 0.06$ is a factor of 2 below their quoted value $\log (\mathrm{TiII})=11.52 \pm 0.06$. On the other hand their upper limits of $\log$ (TiII) $=10.79$ and 10.97 for $\gamma^{2}$ Vel and $\theta$ Car respectively are a factor of two below our measured values of $\log ($ TiII $)=11.13 \pm 0.035$ and $10.97 \pm 0.023$. We note that their $\mathrm{NaI}$ and $\mathrm{CaII}$ measurements are in excellent agreement with other authors (e.g. Welty et al. 1996), and the discrepancy in the TiII results could be due to an incomplete removal of the instrumental background signal which is particularly difficult to measure at these very short wavelengths. The $\mathrm{D} / \mathrm{H}$ ratio depends on the amount of molecular hydrogen. For most of the targets here the relative amount of molecular hydrogen is small and we assume $N(\mathrm{H})=N(\mathrm{HI})$ as well as $\mathrm{D} / \mathrm{H}=\mathrm{DI} / \mathrm{HI}$. For three targets only ( $\theta$ Car, HD 90087 and PG0038+199) that have significant molecular contribution we use the numbers from Table 2 of Linsky et al. (2006) and use $N(\mathrm{H})=N(\mathrm{HI})+2 N(\mathrm{H} 2)$ and $\mathrm{D} / \mathrm{H}=$ $(N(\mathrm{DI})+N(\mathrm{HD})) /(N(\mathrm{HI})+2 N(\mathrm{H} 2))$ instead of $\mathrm{HI}$ and $\mathrm{DI} / \mathrm{HI}$. However we have kept for figure annotations HI and DI/HI.

In Fig. 5 we show the TiII/HI ratio as a function of the $\mathrm{D} / \mathrm{H}$ ratio, and error bars associated with the two quantities. $\mathrm{D} / \mathrm{H}$ error bars are taken from Linsky et al. (2006). It is clear that measurement uncertainties are of the same order of magnitude for both related quantities. This will be the case for most attempted correlations. Fitting such data using standard or ordinary least squares methods can lead to bias in the result. In order to take into account both $x$ and $y$ errors, we have adopted the Orthogonal Distance Regression (ODR) method (also called "total least squares method") for our correlative studies. ODR minimizes the weighed orthogonal distance from the data to the fitted curve. In our case of a simple linear fit of $N$ data pairs $\left(x_{i}, y_{i}\right)$, $i=1, N$ with errors $\sigma x_{i}$ and $\sigma y_{i}$ to the function $y=a x+b$, ODR minimizes the quadratic sum $\Sigma\left(\left(y_{i}-a\left(x_{i}+\mathrm{d} x_{i}\right)-b\right) / \sigma y_{i}\right)^{2}+$ $\left(\mathrm{d} x_{i} / \sigma x_{i}\right)^{2}$, i.e. it adjusts the coefficients a and $\mathrm{b}$ as well as the departures $\mathrm{d} x_{i}$ from the initial $x_{i}$ positions. If the errors on $x$ and $y$ are identical, the summed quantities are proportional to the actual orthogonal distances between the data points and the fitted curve, hence the name of the method. If one of the errors is preponderant, the formula is equivalent to the classical least-square method. We have used the ODRPACK95 package (Boggs et al. 1989) implemented in the IGOR 6.0 software to perform our linear fits. In the case of linear fits, there is a unique solution to the minimization. The resulting weighted minimum distances can be visualized in Figs. 5, 8-10. 
Table 2. Compilation of $\mathrm{D} / \mathrm{H}, \mathrm{D} / \mathrm{O}$, titanium and iron data. Errors bars are quoted in the usual way with negative errors as indices and positive errors as exponants. References of TiII data used in the correlations are: (a) Prochaska et al. (2005), (b) Ellison et al. (2007), (c) this work. Targets in common with Ellison et al. (2007) are marked with an asterix. The column-densities derived by these authors are $\log$ (TiII) $=11.10 \pm 0.02$; $11.31 \pm 0.01 ; 11.12 \pm 0.02 ; 11.05 \pm 0.04 ; 11.07 \pm 0.04$ for $\gamma^{2}$ Vel; $\theta$ Car; $\alpha \mathrm{Cru} ; \beta$ Cen and $\zeta$ Pup respectively and are in agreement with our determinations within uncertainties. $\mathrm{D}, \mathrm{H}, \mathrm{D} / \mathrm{H}, \mathrm{D}(\mathrm{Fe})$ are the values compiled by Linsky et al. (2006), while D, O, D/O are those compiled by Oliveira et al. (2006). The three targets with a very high $\mathrm{D} / \mathrm{O}$ and discussed in Sect. 3.2 are marked with a \#; the first $\mathrm{D} / \mathrm{O}$ value is the published value used in Fig. 9a. The second number is the reduced value used in Fig. 9a (see Sect. 3.2).

\begin{tabular}{|c|c|c|c|c|c|c|c|c|}
\hline Target & $\log (\mathrm{NHI})$ & $\log (\mathrm{DI})$ & $\mathrm{D} / \mathrm{H}(\mathrm{ppm})$ & $\mathrm{DI} / \mathrm{OI} \times 10^{2}$ & $\mathrm{D}(\mathrm{Fe})$ & $\log$ (TiII) & $\mathrm{TiII} / \mathrm{HI} \times 10^{10}$ & Ref \\
\hline SiriusB & $17.6^{0.14}$ & $12.81^{0.09}$ & $16.2^{7.2}$ & $3.9^{0.8}$ & $-1.11^{0.12}$ & & & \\
\hline $36 \mathrm{Oph}$ & $17.85_{0.075}^{0.12}$ & $13.025_{0.01}^{0.09}$ & $15_{2.5}^{2.5}$ & & $-0.65_{0.27}^{0.14}$ & & & \\
\hline$\epsilon$ Eri & $17.875^{0.035}$ & $13.03^{0.03}$ & $14.3^{2.5} 1.6$ & & $\begin{array}{l}0.07^{0.27} \\
-1.1\end{array}$ & & & \\
\hline $31 \mathrm{Com}$ & $17.884_{0.03}^{0.035}$ & $13.19 \begin{array}{l}0.025 \\
0.025\end{array}$ & 20.21 .96 & & $-0.78_{0.1}^{0.1}$ & & & \\
\hline HZ43 & $17.93^{0.03}$ & $13.15^{0.025}$ & $16.6^{1.9}$ & $4.6_{0.5}^{0.5}$ & $-1.21_{0.04}^{0.1}$ & & & \\
\hline Procyon & $18.06_{0.05}^{0.05}$ & $13.26^{0.027}$ & $16_{2}^{1.4}$ & & $-1.24_{0.05}^{0.04}$ & & & \\
\hline$\beta$ Cas & $18.132^{0.05}{ }_{0.025}^{0.025}$ & $13.36_{0.03}^{0.027}$ & $16.9^{2} .6$ & & $\begin{array}{l}0.05 \\
-1.220 .1\end{array}$ & & & \\
\hline G191-B2B & $18.18_{0.09}^{0.025}$ & $13.4_{0.035}^{0.003}$ & $16.6^{4.1}$ & $3.5_{0.4}^{0.4}$ & $-0.58_{0.09}^{0.1}$ & & & \\
\hline$\beta \mathrm{Gem}$ & $18.261_{0.037}^{0.09}$ & $13.43^{0.005}$ & $14.8^{4.2}$ & & $\begin{array}{r}00.09 \\
-1.290 .05 \\
0.05\end{array}$ & & & \\
\hline Capella & $18.239_{0.035}^{0.035}$ & $13.44_{0.02}^{0.03}$ & $15.9^{1.5}$ & $2.6_{1.2}^{1.2}$ & $-1.2_{0.04}^{0.04}$ & & & \\
\hline$\alpha$ Tri & $18.327^{0.035}$ & $13.45^{0.02}$ & $13.3^{2}$ & & $-1.13^{0.04}$ & & & \\
\hline WD0621 376 & $18.7^{0.15}$ & $13.85_{0.045}^{0.055}$ & $14.1_{6}^{\frac{2}{6}}$ & $3.9^{0.6}$ & $-1.21_{0.2}^{0.2}$ & & & \\
\hline WD2211 495 & $18.76_{0.15}^{0.15}$ & $13.94^{0.045} 0.05$ & $15.1_{65}^{6.5}$ & $4_{0 .}^{0.6}$ & $-0.96_{0.16}^{0.2}$ & & & \\
\hline WD1634 573 & $18.85^{0.06}$ & $14.05^{0.025}$ & $15.8^{6.5}$ & $3.5^{0.6}$ & $\begin{array}{r}0.16 \\
-1.220 .09\end{array}$ & & & \\
\hline$\alpha \mathrm{Vir}$ & $19_{0.1}^{0.06}$ & $14.2_{0.1}^{0.025}$ & $15.8_{5.8}^{10.3}$ & $4.2_{2}^{2.3}$ & $-1.29 \begin{array}{l}0.1 \\
0.12\end{array}$ & $10.49 \pm 0.13$ & $32.4{ }_{10}^{14.4}$ & c \\
\hline GD246 & $19.11_{0.025}^{0.1}$ & $14.29_{0.045}^{0.1}$ & $15.1_{1.9}^{5.8}$ & $4.2^{2}{ }_{06}^{2.6}$ & $-1.26_{0.06}^{0.12}$ & & & \\
\hline$\lambda \mathrm{Sco}$ & $19.23_{0.03}^{0.025}$ & $14.11_{0.09}^{0.045}$ & $7.6_{1.1}^{1.9}$ & $1.8_{0.4}^{0.6}$ & $\begin{array}{r}0.06 \\
-1.640 .05 \\
0.05\end{array}$ & & & \\
\hline$\beta$ Cen & $19.63_{0.1}^{0.03}$ & $14.7_{0.2}^{0.07}$ & $11.7_{75}^{7.8}$ & & $-1.16_{0.11}^{0.05}$ & $10.99 \pm 0.04$ & $24 \frac{6.9}{54}$ & $*_{\mathrm{c}}$ \\
\hline$\gamma^{2} \mathrm{Vel}$ & $19.71_{0.026}^{0.1}$ & $15.05_{0.03}^{0.2}$ & $21.8_{2.1}^{2.1}$ & & $-1.21_{0.11}^{0.11}$ & $11.11 \pm 0.035$ & $26.3_{2.3}^{2.5}$ & $*_{\mathrm{c}}$ \\
\hline BD284211 & $19.85^{0.02}$ & 14.990 .025 & $13.9^{2} \mathrm{i}^{\mathrm{I}}$ & $4.7^{0.4}$ & $-1.2^{0.1^{0.11}}$ & $11.08 \pm 0.08$ & $17^{3.4}{ }^{2.3}$ & $\mathrm{a}$ \\
\hline$\alpha$ Cru & $19.85_{0.1}^{0.02}$ & $14.95_{0.05}^{0.025}$ & $12.6_{2.7}^{3.6}$ & & $-1.3_{0.12}^{0.14}$ & $11.12 \pm 0.035$ & $19.5^{3.9} .9$ & $*_{\mathrm{c}}$ \\
\hline$\mu \mathrm{Col}$ & $19.86^{0.015}$ & $14.7^{0.3}$ & $6.9^{6.9}$ & & $\begin{array}{r}0.12 \\
-1.18^{0.02}\end{array}$ & $11.50 \pm 0.02$ & $45.7^{3.3 .3}$ & $\mathrm{c}$ \\
\hline Lan 23 & $19.89_{0.04}^{0.25}$ & $15.23_{0.065}^{0.1}$ & $\begin{array}{l}01.817 .4 \\
21.9\end{array}$ & $3.2_{1.6}^{1.6}$ & $\begin{array}{r}0.02 \\
-1.31_{0.07}^{0.07}\end{array}$ & & & \\
\hline$\zeta$ Pup & $19.96_{0.026}^{0.024}$ & $15.11_{0.06}^{0.065}$ & $14_{2.3}^{2.1}$ & & $-1.28 \begin{array}{r}0.17 \\
0.07\end{array}$ & $11.16 \pm 0.06$ & $16.6_{2.5}^{2.9}$ & $*_{\mathrm{c}}$ \\
\hline TD1 32709 & $20.03^{0.1}$ & $15.3^{0.05}$ & $18.6^{5.3 .3}$ & $7.59 ; 3.75^{2.17}$ & $-1.53^{0.14}$ & & & \# \\
\hline WD1034+001 & $20.07_{0.07}^{0.1}$ & $15.4_{0.07}^{0.05}$ & $21.4^{5.3}$ & $6.31 ; 3.15_{1.79}^{1.76}$ & $-1.42_{012}^{0.14}$ & $11.1 \pm 0.2$ & $10.7_{41}^{6.7}$ & $\mathrm{b \#}$ \\
\hline $\mathrm{BD}+393226$ & $20.08 \begin{array}{r}0.07 \\
0.09\end{array}$ & $15.15_{0.05}^{0.07}$ & $11.7^{4.5} 3.1$ & $5.62 ; 2.81$ & $-1.38_{0.11}^{0.12}$ & $11.49 \pm 0.08$ & & $\mathrm{b \#}$ \\
\hline Feige 110 & $20.14^{0.065}$ & $15.47^{0.03}$ & $21.38_{3}^{3.1}$ & $2.6^{0.5}$ & & $11.59 \pm 0.03$ & $28.2^{4.9}$ & $\mathrm{a}$ \\
\hline$\iota$ Ori & $20.16_{0.07}^{0.11}$ & $15.3_{0.05}^{0.043}$ & $14.13_{2.8}^{2.8}$ & $3.5_{0.8}^{0.5}$ & $-1.41_{0.16}^{0.21}$ & $11.31 \pm 0.03$ & $14.1_{2.1}^{2.2}$ & $\mathrm{a}$ \\
\hline$\gamma$ Cas & $20.16^{0.08}$ & $15.15^{0.05}$ & $9.8^{2.3 .8}$ & $2.5^{0.4}$ & $\begin{array}{r}1.530 .14 \\
-1.14\end{array}$ & & & \\
\hline$\delta$ Ori & $20.19^{0.1} 0.13$ & $15.06^{0.06}$ & $\begin{array}{l}7.41 .2 \\
0.9\end{array}$ & $2.5^{0.4}$ & $\begin{array}{r}0.18 \\
-1.56^{0.04}\end{array}$ & $11.15 \pm 0.04$ & $9.1_{1}^{1.1}$ & $\mathrm{a}$ \\
\hline$\theta$ Car & $20.28_{0.1}^{0.03}$ & $14.98_{0.18}^{0.04}$ & $5_{3 .}^{2.9}$ & & $\begin{array}{r}0.04 \\
-1.540 .11\end{array}$ & $11.31 \pm 0.023$ & $11.2^{12.9}$ & $*_{\mathrm{c}}$ \\
\hline$\epsilon$ Ori & $20.4_{0.1}^{0.08}$ & $15.25^{0.05}$ & $6.3^{3.4}$ & $1.9_{0.3}^{0.3}$ & $-1.7^{0.14}$ & $11.4 \pm 0.03$ & $10.0_{10}^{2.3}$ & $\mathrm{a}$ \\
\hline PG $0038+199$ & $20.48_{0.04}^{0.1}$ & $15.76_{0.04}^{0.05}$ & $19.1_{2.6}^{1.5}$ & $2.4{ }_{0.5}^{0.3}$ & $\begin{array}{l}0.12 \\
-1.51_{0.04}^{0.05}\end{array}$ & & & \\
\hline JL9 & $20.78_{0.05}^{0.04}$ & $15.78_{0.06}^{0.04}$ & $10_{19}^{1.9}$ & $1.9_{0.8}^{0.5}$ & $-1.54_{0 .}^{0.04}$ & $11.88 \pm 0.1$ & $13.2_{29}^{3.8}$ & $\mathrm{c}$ \\
\hline HD 195965 & $20.95^{0.025}$ & $15.88_{0.07}^{0.06}$ & $\begin{array}{r}8.91_{1.9}^{1.9} \\
8.6\end{array}$ & $1.3^{0.3}$ & $\begin{array}{r}-1.59^{0.1} 03 \\
0.03\end{array}$ & $12.02 \pm 0.02$ & $11.7^{2.8}$ & a \\
\hline LS1274 & $20.98_{0.04}^{0.025}$ & $15.86_{0.09}^{0.07}$ & $7.6^{1.9}{ }_{1.9}^{1.6}$ & $1.8_{0.5}^{0.3}$ & $\begin{array}{r}-1.620 .08 \\
0.08\end{array}$ & $11.68 \pm 0.04$ & $5.2^{0.8} 8^{0.8}$ & $\mathrm{c}$ \\
\hline HD 191877 & $21.05^{0.05}$ & $15.94^{0.11}$ & $7.8^{1.9} .4$ & $2.5^{1}$ & $-1.55^{0.05}$ & $12.24 \pm 0.02$ & $15.5^{1.9}$ & $\mathrm{a}$ \\
\hline HD 41161 & $\begin{array}{l}0.05 \\
21.08_{0.09}^{0.09}\end{array}$ & $16.41_{0.05}^{0.06}$ & $21.4_{4.5}^{5.5}$ & & & $12.28 \pm 0.04$ & $15.8_{3.3}^{1.7}$ & $\mathrm{~b}$ \\
\hline HD 53975 & $21.14_{0.06}^{0.09}$ & $16.15_{0.07}^{0.05}$ & $10.2^{2.4}$ & & & $12.13 \pm 0.04$ & $9.8_{1.5}^{1.7 .3}$ & $\mathrm{~b}$ \\
\hline HD 90087 & $21.22_{0.05}^{0.06}$ & $16.16_{0.06}^{0.06}$ & $8.7^{1.7}$ & $1.7_{0.4}^{0.4}$ & $-1.45_{0.05}^{0.05}$ & & & \\
\hline
\end{tabular}

The ODRPACK95 package works for symmetric errors, i.e. equal positive and negative error intervals. If there are substantial differences between the positive and negative errors, the data point weight depends not only on the distance to the fitted line, but also on which side it is located with respect to it, with lower (resp. smaller) weight if it is located on the large (resp. small) uncertainty side. In order to take into account non symmetric errors we devised and we use the following iterative method: we first calculate the average (mean of positive and negative) error for Xs (resp. Ys) and run the ODR linear fit for those mean errors. A simple routine then identifies which data points are above or below (resp. to the left or to the right of) the fitted straight line and derives from their locations which error (the positive or the negative one) is appropriate. A second fit is then activated with those new appropriate errors. This process is repeated until convergence. For linear fits and a relatively small numbers of points, the convergence is immediate. The method is illustrated in Fig. 5. We show two data points for which the appropriate errors are of opposite signs. For all our regressions we show the resulting orthogonal distances between data points 

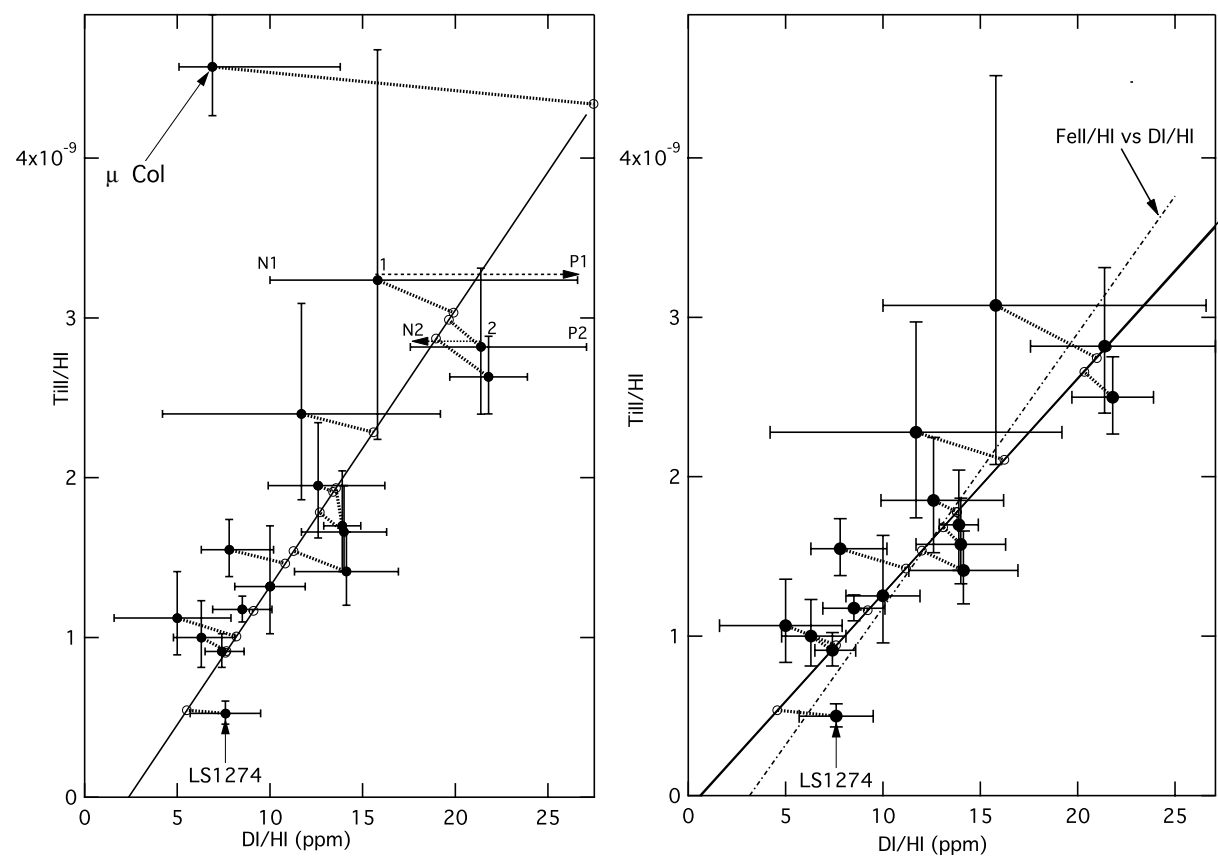

Fig. 5. Ionized titanium to neutral hydrogen column ratio vs. DI/HI with (left) and without (right) the star $\mu$ Col. The method used to take into account non symmetric error bars for the abscissa is illustrated in the left figure. For the data point 1 (resp. 2), because it is located on the left (resp. right) side of the fitted line, the selected error bar is P1 (resp. N2). The same method is applied for the errors in the ordinate parameter. The corresponding linear fit for FeII is shown as a dotted line in the right figure.

and the corresponding adjusted locations along the fitted line. All quoted uncertainty intervals on the parameters are $1 \sigma$ and calculated using the error bars from the last fit after convergence.

We list in Table 3 two diagnostics for the correlation, the Spearman index and the slope interval for the fitted linear relationship. Note that the Spearman's rank correlation statistic does not take into account error bars, while the error on the fitted parameters does, which means that in some cases they may give different results.

\subsection{Correlations of Till and Fell abundances with $\mathrm{D} / \mathrm{H}$ and $\mathrm{D} / \mathrm{O}$}

Visual inspection of Fig. 5 shows that there is a positive correlation between TiII/HI and DI/HI, with the noticeable exception of the star $\mu \mathrm{Col}$. The peculiarity of this target is already visible in Figs. 4, 6, 7 from Linsky et al. (2006) for FeII and SiII. For all three species the abundance is significantly above that of the main trend. To a lesser extent a second target also seems out of the main relationship, namely LS1274. In order to check the influence of the HI column on this result, we have displayed the TiII columns as a function of DI in Fig. 6. $\mu \mathrm{Col}$ is clearly an outlier and LS1274 is also somewhat out of the main trend. We have investigated possible reasons for the peculiarity of $\mu \mathrm{Col}$, but could not find anything in the literature. On the other hand, we have seen in the previous section that there is strong evidence against an interstellar origin of the broad line detected towards this star, on the basis of the disagreement between the 3384 and 3242 lines. Interestingly, using the narrow line only moves the star into the main trend. Therefore we performed linear fits to the data, with and without $\mu \mathrm{Col}$. On the other hand we have chosen to maintain LS1274. Its absence does not make any noticeable overall difference.

The Spearman's rank correlation statistic is 0.84 (resp. 0.59) without (resp. with) $\mu \mathrm{Col}$ (15 (resp. 16) points) allowing us to reject the null correlation hypothesis at better than 99.97 (resp. 98)\%. The parameters for the slope of the linear fit are $(1.35 \pm 0.22)$ and $(1.66 \pm 0.29) \times 10^{-10}$ respectively (see Table 3 ). Thus, while clearly $\mu \mathrm{Col}$ modifies the fitted straight line, in both cases there is a positive correlation since the most probable gradient value is about 6 times the $1 \sigma$ uncertainty on this slope. The reduced $\chi^{2}$ is 1.3 and 1.1 in both cases, thus there is possibly some intrinsic variability in addition to the positive correlation. The intercept is negative but compatible with zero without $\mu$ Col.

The results for TiII, keeping the same methods and adding the 4 new Ellison et al. targets, are not noticeably different from those based on our own data and the Prochaska et al. data only (see Table 3). However, the presently derived slope is significantly different from the one found by Ellison et al. (2007). There are two reasons for this disagreement. (i) We believe their quoted parameters for the linear fit correspond to equal weights for all data points because we find the same parameters using their listed data and no specific weights. (ii) The fitting method is not the same. Using error bars in $X$ and $Y$ leads to different results. (iii) The data sets are not the same.

In order to compare in the most reliable way the TiII results with those of FeII, we have also applied the same fitting procedure to FeII data, i.e. using the linear iron abundances (and not the depletion index) and the ODR method. The data and results are shown in Fig. 7. It can be seen that the dispersion of iron abundances appears significantly greater than that of titanium. Three targets are especially clearly discrepant, with G191-B2B being in the most disagreement. Despite this dispersion we have fitted the iron abundance vs. D/H distribution without excluding any of the targets. We will come back to this important point later. Fit parameters are listed in Table 3.

Such a positive correlation could be due to random or systematic errors in the determination of HI (e.g. Hébrard \& Moos 2003) since HI appears in both related quantities and is by far 
Table 3. Correlation parameters. LHW = this paper, PTH05 = Prochaska et al. (2005), EPL07 = Ellison et al. (2007), Lin06 = Linsky et al. $(2006)$, O106 = Oliveira et al. (2006)

\begin{tabular}{|c|c|c|c|c|c|c|}
\hline $\begin{array}{l}\text { Correlation } \\
y=a+b x\end{array}$ & $\begin{array}{c}\text { Targets } \\
x=\text { list intersection }\end{array}$ & Data points & $a \pm 1 \sigma$ & $\begin{array}{c}b \pm 1 \sigma \\
\text { correlation }\end{array}$ & Spearman test & $\chi^{2} / N$ \\
\hline (TiII/HI) vs. (DI/HI) & LHW + PTH05 & 16 & $(-3.6 \pm 2.9) \times 10^{-10}$ & $(1.66 \pm 0.29) \times 10^{-4}$ & $98 \%$ & 1.3 \\
\hline (TiII/HI) vs. (DI/HI) & Same without $\mu \mathrm{Col}$ & 15 & $(-0.8 \pm 2.3) \times 10^{-10}$ & $(1.35 \pm 0.22) \times 10^{-4}$ & $99.97 \%$ & 1.1 \\
\hline (TiII/HI) vs. (DI/HI) & Same + EPL07 (no $\mu \mathrm{Col})$ & 19 & $(-0.6 \pm 2.32) \times 10^{-10}$ & $(1.29 \pm 0.21) \times 10^{-4}$ & $99.5 \%$ & 1.0 \\
\hline (FeII/HI) vs. (DI/HI) & Table 2 Lin06 & 38 & $(-1.56 \pm 0.76) \times 10^{-2}$ & $(4.96 \pm 0.65) \times 10^{+3}$ & $99.7 \%$ & 1.6 \\
\hline (TiII/HI) vs. (DI/OI) & $(\mathrm{LHW}+\mathrm{PTHO} 5) \times(\mathrm{Oli06})$ & 10 & $(-1.24 \pm 3.29) \times 10^{-10}$ & $(6.05 \pm 1.45) \times 10^{-8}$ & $98 \%$ & 2.7 \\
\hline (TiII/HI) vs. (DI/OI) & Same+EPLO7 & 12 & $(-2.07 \pm 3.46) \times 10^{-10}$ & $(6.46 \pm 1.50) \times 10^{-8}$ & $97 \%$ & 2.3 \\
\hline$(\mathrm{Fe} / \mathrm{Fe} 0)$ vs. (DI/OI) & (Table 2 Lin06) x (Oli06) & 23 & $(3.5 \pm 5.6) \times 10^{-3}$ & $1.28 \pm 0.22$ & $99.6 \%$ & 1.3 \\
\hline$(\mathrm{Fe} / \mathrm{Fe} 0)$ vs. $(\mathrm{DI} / \mathrm{OI})$ & Same with 3 D/O \%2 (see text) & 23 & $(-0.5 \pm 6.6) \times 10^{-3}$ & $1.49 \pm 0.26$ & $99.95 \%$ & 0.7 \\
\hline (TiII/DI) vs. (LogNH) & LHW + PTH05 (no $\mu \mathrm{Col})$ & 15 & $(5.9 \pm 2.5) \times 10^{-4}$ & $(-2.34 \pm 1.24) \times 10^{-5}$ & $25 \%$ & 1.46 \\
\hline (TiII/DI) vs. $(\operatorname{LogNH})$ & same + EPL07 & 19 & $(7.6 \pm 1.8) \times 10^{-4}$ & $(-3.2 \pm 0.9) \times 10^{-5}$ & $64 \%$ & 1.8 \\
\hline
\end{tabular}

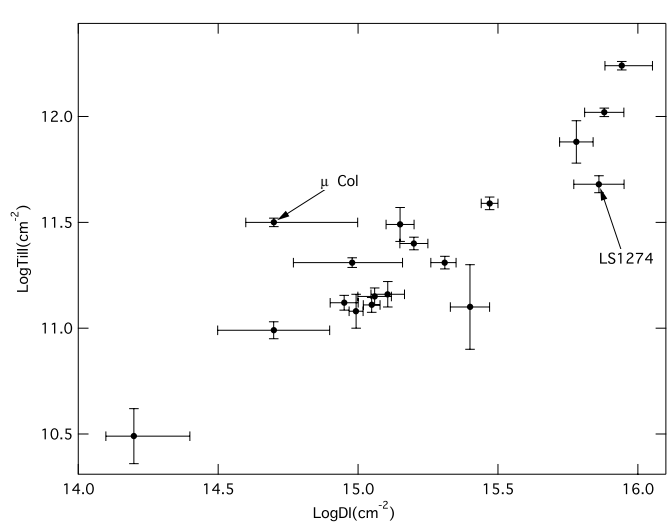

Fig. 6. Titanium vs. deuterium columns. The star $\mu \mathrm{Col}$ is clearly an "outlier".

the most difficult measurement. Indeed, changing HI may move the respective data point along the straight line fit. This is also the case for correlations between metal/O ratios with $\mathrm{D} / \mathrm{O}$ ratios. Consequently, among our tests we have searched for a correlation between the abundance of titanium (TiII/H) and the neutral deuterium to neutral oxygen (DI/OI) ratio. This study is restricted to titanium targets for which the OI column is available in the literature. Interestingly, although the number of data points used is smaller (10 points), there is clearly a trend, as can be seen in Fig. 8. Quantitatively, the Spearman test rejects the absence of positive correlation at better than $98 \%$, and the TiII/HI vs. DI/OI slope is found to be $6.0 \pm 1.4 \times 10^{10}$ (see Table 3). Such a trend using four independent quantities is a definite sign of a positive correlation between metals and deuterium. Adding the two Ellison et al. targets for which D/O is available does not significantly change this conclusion. The correlation parameters for the 12 point correlation are listed in Table 3.

This result led us to correlate in a similar way the iron $(\mathrm{Fe})$ depletion with the DI/OI ratio. Instead of using a logarithmic scale, we have used the linear ratio between the abundance of FeII and the solar abundance from Asplund et al. (2005). Here the number of available measurements is higher (24 lines-ofsight, see Table 2).

Among these three data points there is an extreme "outlier", G191-B2B. The gas towards this target is almost fully ionized, and as discussed in Sect. 3.3.1 there may be a strong bias in the evaluation of iron depletion because iron is less rapidly ionized than $\mathrm{H}$ (see Linsky et al. 2006). We have chosen to exclude this target from our correlation fit, and we will come back to the ionization problem later and justify this choice. This leaves us

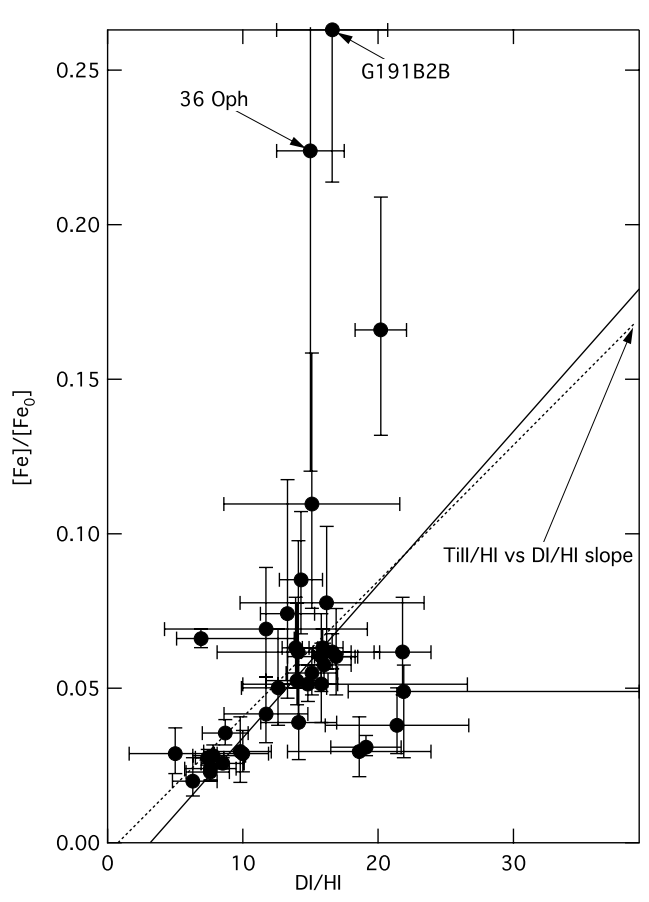

Fig. 7. Ionized iron abundance normalised to $(\mathrm{Fe} / \mathrm{H})_{0}=-4.55$ (Asplund et al. 2005) vs. DI/HI. This figure is identical to Fig. 3 from Linsky et al. (2006) except for the linear scale. The best fit linear correlation using the Orthogonal Distance Regression (ODR) is shown as a solid line, and for comparison the titanium gradient obtained with the same method (Fig. 5) is shown as a dashed line after the appropriate scaling to obtain a similar ordinate for $\mathrm{D} / \mathrm{H}=20$. It can be seen that the dispersion is significantly larger for FeII than for TiII. Lines-of-sight filled with strongly ionized gas (G191-B2B, 36 Oph) are positive extreme "outliers", while those corresponding to very large columns tend to produce negative "outliers".

with 23 sight-lines, and the results are shown in Fig. 9. Note that G191-B2B would be completely out of the figure, with a $\mathrm{Fe} / \mathrm{Fe} 0$ of 0.26. As seen with titanium in Fig. 7, the data point distribution reveals a clear positive correlation. Three points show a rather large deviation from the mean curve and seem to have a much higher $\mathrm{D} / \mathrm{O}$ compared to the other targets. Those very distant targets are indeed the only three targets for which very high D/O ratios have been claimed: WD 1034+001, BD +393226 and TD1 32709 (Oliveira et al. 2006). However, toward those targets the $\lambda 974 \AA$ OI transition has not been used in the OI column determination, or was not significantly detected. Not using this transition could lead to underestimation of $N(\mathrm{OI})$ by factors of 
$\simeq 2$, as it has been shown in the cases of Feige 110 (Hébrard et al. 2005) and LSE 44 (Friedman et al. 2006). Using this transition, the OI column density toward BD +39 3226 is also multiplied by a factor of 2.6 (Oliveira et al. 2006). Thus, as suggested by Fig. 9, the D/O measurements might be overestimated by a factor of 2 to 3 for these three lines of sights.

We have thus performed two analyses: firstly using the 3 high DI/OI values from the strong line fitting, and secondly after division by a factor of 2 of the DI/OI ratios for only these three distant stars, hypothesizing that a correction similar to the Feige 110 re-evaluation is likely to be necessary. When this allowance for correction of line saturation effects is included, the three corresponding data points all move back closer to the main stream of data points. We have also performed a similar linear fit, excluding the three targets instead of revising their DI/OI and found results very similar to those we show here. The two fits are displayed in Fig. 9 and the linear fit parameters before and after correction are given in Table 3. The Spearman test rejects the absence of correlation at better than $99.6 \%$ and $99.99 \%$ in the first and second case respectively. The gradient is somewhat steeper after the correction for the three high columns, namely $1.49 \pm$ 0.26 instead of $1.28 \pm 0.22$.

The TilI and FeII positive correlations with $\mathrm{D} / \mathrm{O}$ demonstrate three important points. Firstly, potential errors in the measured values of $\mathrm{HI}$ as the source of a link between $\mathrm{D}$ and metals in the gas phase is a hypothesis that can now be excluded as the main origin of the deuterium-metal correlation. If errors in the measurement of HI were the case, we would simply have randomly distributed points. Secondly, the D/O ratio, despite its different evolution as a function of the gas column compared to DI/HI, is shown to vary in a specific way, and shows a link to the metal abundance. Third, D/O cannot be linked to metal depletion principally through astration effects, since this would produce the opposite trend, with $\mathrm{D} / \mathrm{O}$ being anti-correlated with the degree of astration and thus with metal abundance.

\subsection{Gradient comparisons}

\subsubsection{Comparisons between titanium and iron}

It is interesting to compare the variation amplitude of titanium and iron. Titanium is strongly refractory and one expects a higher amplitude of the gas-phase abundance variation, as argued by Ellison et al. (2007). Figure 5 shows the FeII vs. DI/HI gradient from Fig. 7 superimposed on the TiII vs. DI/HI gradient. Contrary to expectations based on condensation temperatures, TiII varies slightly less than FeII, although as shown in Table 3 and taking into account the two error bars, the two gradient intervals nearly overlap. In the case of DI/OI the two gradients are compatible, but the TiII vs. DI/OI gradient is less precisely defined than for DI/HI.

We believe that the absence of a stronger gradient for TilI is not an inevitable concern with respect to the deuterium depletion hypothesis of Linsky et al. (2006) and that the observed superiority or similarity of the iron slope can be explained by ionization effects. Linsky et al. (2006) have already discussed the potential impact of differential ionization of FeII and HI on the FeII/DI ratio variation. The ionization potential of FeII is $16.2 \mathrm{eV}$, and as a consequence hydrogen may ecome ionized by the radiation field while FeII remains only slightly affected. The case of G191-B2B, the extreme outlier, is typical. From their analysis of high resolution HST-GHRS spectra, Lemoine et al. (1999) inferred the existence of two main clouds along the LOS to the hot white dwarf. For the main HI cloud (B) the FeII/HI ratio is

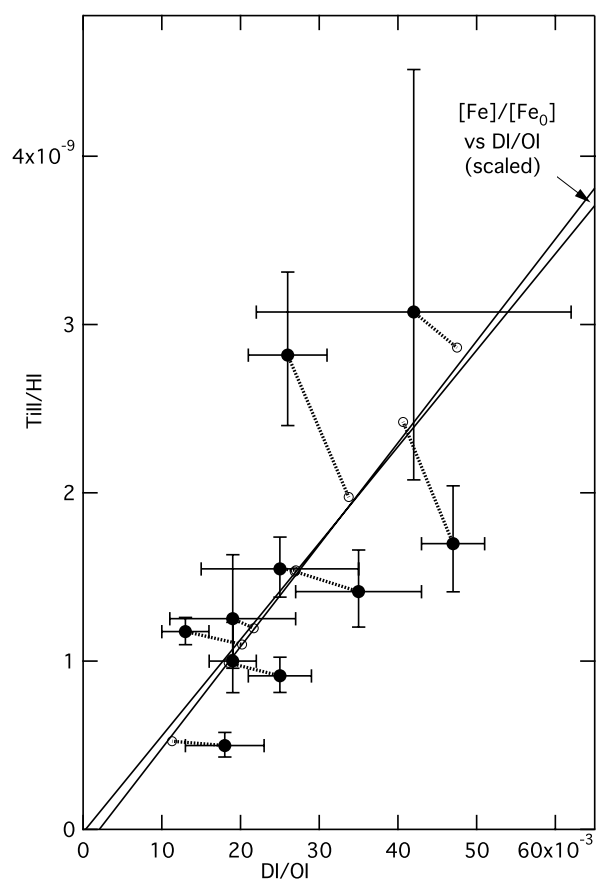

Fig. 8. Left: ionized titanium vs. DI/OI. The corresponding linear fit for FeII (Fig. 5) is shown for comparison.

$0.16 \times 10^{-5}$ while for the second, strongly ionized cloud (A), the same ratio is $2.25 \times 10^{-5}$, i.e. 13,14 times above cloud $\mathrm{B}$. The existence of the ionized fraction contained in cloud $\mathrm{A}$ has the effect of increasing the overall ratio (for $\mathrm{A}+\mathrm{B}$ ) by a factor of more than 3 compared to cloud $\mathrm{A}$ alone. This is a very strong effect, and it is clear from Fig. 7 that lines-of-sight filled with significantly ionized gas, such as G191-B2B or 36 Oph have a systematically higher singly ionized iron abundance, that probably does not reflect the actual iron abundance. As a consequence it is likely that a high ionization degree, a phenomenon expected to be present in shock- and photo-heated regions, i.e. in regions with the strongest evaporation and deuterium release from grains, also tends to increase the FeII/HI ratio. In other words, in the absence of precise corrections for the gas ionized fraction, iron release in the gaseous phase and apparent abundance increase due to ionization balance effects act in a cumulative way. This tends to increase the FeII/H ratio and the larger gradient for FeII may result from this trend. As we explained earlier G191-B2B is an extreme case and we have removed it from the correlation with DI/OI shown in Fig. 9, which has the effect of decreasing the slope. Probably other remaining targets such as WD2211-495 have an effect on the slope of the linear fit. G191B2B is also the most discrepant point for the FeII/HI vs. $\mathrm{DI} / \mathrm{HI}$ correlation, but because there are two times more targets for this correlation it is not isolated, and this is why we have kept it. Looking at Fig. 7 it is clear that three data points including this star tend to increase the gradient of the correlation. These ionization effects are absent in the case of TiII, due to the similarity of the potentials. This is why we believe that the absence of the expected stronger gradient for titanium compared to iron is due to an overestimate of the latter.

\subsubsection{Comparison between the titanium and iron gradients vs. D/H and vs. D/O}

While the positive correlation between the metal abundance and $\mathrm{D} / \mathrm{O}$ can not be explained by astration (it would be a negative 

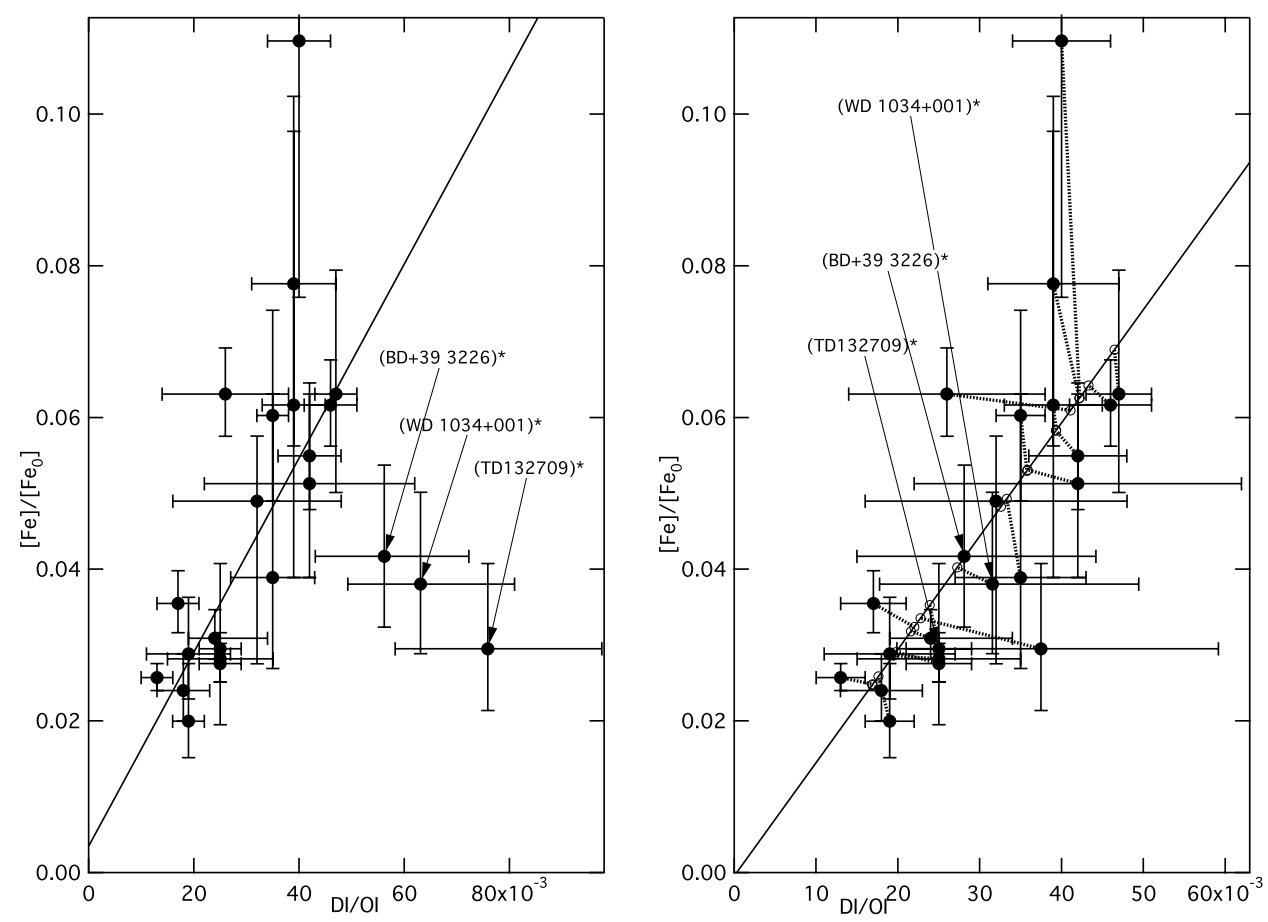

Fig. 9. Iron gaseous abundance (normalised to the Asplund et al. abundance) and deuterium to oxygen ratio. a) Published DI/OI results. b) After division by a factor of 2 of the three DI/OI ratios for the three distant stars (see text).

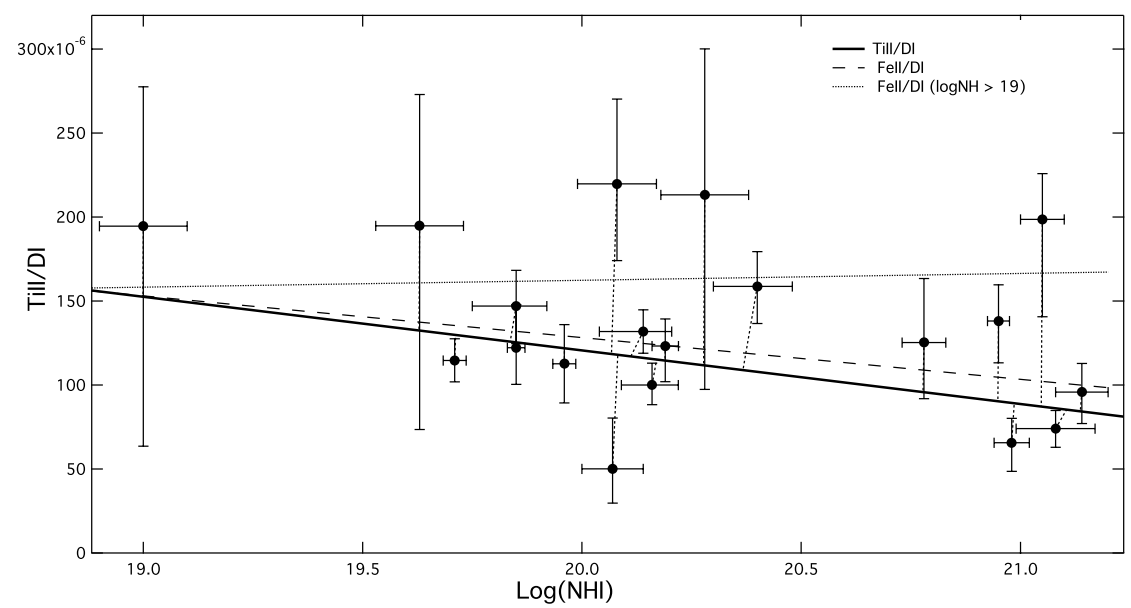

Fig. 10. TiII/DI ratio as a function of the HI column: all available measurements. The linear fit using the Orthogonal Distance Regression method is shown as a solid line. The slopes found by Linsky et al. (2006) for the FeII/DI ratio are shown as dashed and dotted lines (resp. all data, and data with $\log N(\mathrm{HI}) \geq 10^{19} \mathrm{~cm}^{-2}$ ).

trend), it may be well explained, as for D/H (Linsky et al. 2006), by the depletion hypothesis. Here we compare the two gradients obtained from the previous correlations of TiII and FeII with $\mathrm{D} / \mathrm{H}$ and $\mathrm{D} / \mathrm{O}$.

Along with deuterium and metals that evaporate from grains and are released in the gaseous phase, it is reasonable to expect some simultaneous release of oxygen. However the relative increase of the OI concentration due to this release is much smaller than for DI, because the oxygen abundance is the gaseous phase is much higher than for deuterium. Also, data show a quasiconstancy of the OI/HI ratio, showing that an increase due to dust evaporation is negligible. Therefore, in the depletion scenario, for a given increase of the metal gaseous abundance, i.e., of dust evaporation, DI/OI should increase in the same proportions as DI/HI. Figures 5, 7-9 and Table 3 show that this is the case globally, i.e. the metal abundance, the $\mathrm{D} / \mathrm{H}$ ratio and the
$\mathrm{D} / \mathrm{O}$ ratio all vary by a factor of $3-3.5$, and there are significant linear correlations between metals and $\mathrm{D} / \mathrm{H}$ on one hand, metals and $\mathrm{D} / \mathrm{O}$ on the other. However, there is no simple proportionality between the three quantities, since some lines of sight with very high $\mathrm{D} / \mathrm{H}$ ratios do not present correspondingly high $\mathrm{D} / \mathrm{O}$ values (Hébrard et al. 2005).

It is striking that error intervals for the fit parameters allow (or nearly allow) a simple proportionality between $\mathrm{Ti}$ or $\mathrm{Fe}$ and $\mathrm{D}$ ( the linear fit in this case goes through zero). This implies that $\mathrm{D}$ increases as rapidly as metals. In the framework of the deuterium depletion scenario of Draine (2004) and Linsky et al. (2006), one would expect a preferential release of deuterium from PAHs and the carbonaceous envelopes of grains, whereas metals $(\mathrm{O}$ and $\mathrm{Fe})$ are instead preferentially removed from the cores of interstellar grains, and therefore a strict proportionality is somewhat surprising. 
The comparison between the residuals for TilI/HI vs. D/H $\left(\chi^{2} / N=1.3\right.$ and 1.1 , see Table 3$)$ and $\mathrm{D} / \mathrm{O}\left(\chi^{2} / N=2.7\right.$ and 2.3$)$ shows that the fit is of significantly better quality vs. $\mathrm{D} / \mathrm{H}$ than vs. D/O. We believe this is due to the fact that in the former case $N(\mathrm{H})$ appears in the two correlated quantities. As a consequence, measurement errors on $\mathrm{H}$ tend to produce dispersion along the fitted line, that does not increase the $\chi^{2}$. This shows that a fraction of the correlation between TiII/HI and D/H is due to uncertainties on HI columns. In the latter case of the correlation with $\mathrm{D} / \mathrm{O}$, measurement errors in $\mathrm{H}$ bring a dispersion along the ordinate axis that appear in the $\chi^{2}$. Such effects for iron are not seen because the residuals are dominated by the ionisation biases that introduce a strong dispersion. Especially, we have removed G191B2B for the correlation with D/O and kept it for $\mathrm{D} / \mathrm{H}$, which explains the higher $\chi^{2} / N$ for $\mathrm{D} / \mathrm{H}$.

\subsubsection{Correlation between titanium abundances and column-densities}

We have searched for deviations from proportionality and for a potential link between the titanium to deuterium abundance ratio and the HI column. Linsky et al. (2006) have showed evidence for an increase of the DI/FeII ratio along with the HI column. More precisely, there seems to be an increase from very low columns $\left(10^{18} \mathrm{~cm}^{-2}\right)$ to columns of the order of $10^{19-19.5} \mathrm{~cm}^{-2}$ and constancy above $\log (\mathrm{NH}) \simeq 19.5 \mathrm{~cm}^{-2}$. They interpreted this trend as evidence for a quasi proportionality of $\mathrm{D}$ and Fe abundances in general, and attributed the low to medium column increase as being due to significant ionisation of the diffuse gas close to the Sun, an effect similar to what we have invoked for the Ti vs. Fe comparison. As discussed by the authors and previously in this paper for HI, in ionized gas D may be more easily ionised than FeII, leading to an overestimate of the abundance of FeII at low columns within the Local Bubble.

In the case of TiII, ionisation effects are not supposed to have any contribution due to the coincidence between the two ionisation potentials of TiII and HI (or DI). It is therefore particularly interesting to perform the same correlation test as the one done by Linsky et al. but for TiII instead of FeII. Figure 10 shows the TiII/DI ratio as a function of $\log N(\mathrm{HI})$. Similarly to the Linsky et al. (2006) results for FeII, the smallest HI columns seem to correspond to the highest TiII/DI ratio. While the trend is not as clearly defined as in the correlation analyses in the previous sections, with a Spearman rejection of null correlation at about $75 \%$ probability only (and a negative result if we do not include the high column targets of Ellison et al. 2007, see Table 3), the least-squares linear fit with the ODR method taking into account error intervals on TII/DI and $\log N(\mathrm{HI})$ demonstrates a negative slope at better than $3.5 \sigma \mathrm{s}$. However, the slope is very strongly dependent on the three points with high columns and small error bars and more or better data are needed to assess this result.

The sign of this inferred TiII/DI gradient cannot be explained by less astration for high columns, i.e. far from the Sun, since it would contradict the increase of $\mathrm{OI} / \mathrm{HI}$ at large columns found by Andre et al. (2003) and more recently by Oliveira et al. (2006). Tentatively, the sign of the slope may correspond to preferential evaporation of deuterium compared to titanium at the highest columns, i.e. in very dense and colder regions which become preponderant along very distant lines-of-sight. In other words, D in this case is released into the ISM before metals, and only in warmer and more tenuous media are the release rates identical.

Superimposed on the Tii/DI vs. $\log N(\mathrm{HI})$ fitted relationship are shown the corresponding results of Linsky et al. (2006) for FeII. Ordinates have been multiplied by a constant, and we are interested here in the slope only. Our results that correspond to $\log N(\mathrm{HI}) \geq 19$ should be compared to the flat curve calculated by these authors for targets with $\log N(\mathrm{HI}) \geq 19.3$.

As we already mentioned, titanium data are very appropriate here because of the absence of ionization effects that may introduce biases. However there is a lack of measurements with $\log N(\mathrm{H})$ smaller than $10^{19} \mathrm{~cm}^{-2}$ to confirm unambiguously a trend.

\section{Discussion and conclusions}

We have observed nine southern hemisphere $\mathrm{D} / \mathrm{H}$ target stars at high spectral resolution and derived the column-densities of interstellar ionised titanium towards those targets. A comparison between the $\mathrm{D} / \mathrm{H}$ ratio and the titanium depletion reveals a clear correlation and reinforces the result of Prochaska et al. (2005) and the more recent result of Ellison et al. (2007). Our work extends the correlation over a broader range of interstellar HI columns, namely more than two orders of magnitude, showing that the trend extends down to $\log N(\mathrm{HI})=19.0 \mathrm{~cm}^{-2}$. We also make use of the orthogonal distance regression method for our linear fits, in order to take into account errors in quantities both in abscissa and ordinate.

One star is far out of the correlation, $\mu \mathrm{Col}$. This target is already strongly discrepant in correlations shown by Linsky et al. (2006) between D/H and iron and silicon abundances. We have shown evidence here for a non-interstellar origin of the broadest absorption feature and note that excluding this absorption would bring the star back to the main trend.

We have compared both the $\mathrm{Ti}$ and Fe depletions with the $\mathrm{DI} / \mathrm{HI}$ ratio, but also with the DI/OI ratio, when available. For both metals there is a clear relationship also with DI/OI, which demonstrates that the metal abundance- $\mathrm{D} / \mathrm{H}$ correlation is not primarily due to uncertainties in HI column measurements, and that $\mathrm{D} / \mathrm{O}$ is also correlated with metal depletions in the ISM.

The ionized titanium correlation gradient is found to be similar to or slightly smaller than the corresponding gradient for ionized iron. Ellison et al. (2007) have emphasized that given its high condensation temperature one would expect the titanium gradient to be the highest. We have argued here that ionization effects do affect FeII/HI and result in an overestimate of the gradient, while the titanium abundance is unaffected due to the nearly exact coincidence between TiII ad HI ionization potentials. Thus we believe that the slopes measured for both metals are not a concern with respect to the depletion hypothesis as the source of deuterium abundance variations. Further work is needed to correct for the ionization and obtain a more reliable comparison between the different metal-deuterium relationships.

There is some (although weak) evidence that the TilI/DI ratio decreases towards high column densities. This may signify that D is preferentially released into the ISM (when compared to titanium) in the dense regions that are predominant along distant lines-of-sight. It is important to investigate further this potential relationship, because, if confirmed, it would be a first indication of the different sources of interstellar D and metals. Recording more titanium data along shorter lines-of-sight is essential. TiII, that does not suffer ionisation biases, is an ideal tracer here.

It remains to explain why very high $\mathrm{D} / \mathrm{H}$ ratios do not have corresponding very high D/Os, as already argued by Hébrard et al. (2005). This is reinforced here by our findings (Sect. 3.2 and Fig. 9a) that the three high $\mathrm{D} / \mathrm{O}$ values found by Oliveira et al. (2006) may be overestimated. Additionally, Oliveira \& Hébrard (2006) derive a high D/H for a very distant sightline while one would expect in this case a very strong depletion. 
Certainly some of the physical processes are not yet understood. On the other hand, since very discrepant points appear in the correlation with $\mathrm{D} / \mathrm{H}$, but none with $\mathrm{D} / \mathrm{O}$, the high $\mathrm{D} / \mathrm{H}$ ratios may be a consequence of underestimated $\mathrm{H}$ columns.

Assuming proportionality between metals and D depletion, one can derive a mean $\mathrm{O} / \mathrm{H}$ ratio from the ratio of the $\mathrm{Ti} / \mathrm{H}$ vs. $\mathrm{D} / \mathrm{H}$ and $\mathrm{Ti} / \mathrm{H}$ vs. D/O slopes (or similarly for Fe). Using TilI one finds (Table 3) $6.05 \times 10^{-8} / 1.35 \times 10^{-4}=448$ ppm using our targets and the Prochaska et al. targets, and $6.46 \times$ $10^{-8} / 1.29 \times 10^{-4}=501 \mathrm{ppm}$ using all targets. Using FeII one finds $1.49 / 4.96 \times 10^{3}=300 \mathrm{ppm}$. There must be some significance to this difference that remains to be elucidated. These two values are respectively higher and lower than the mean value of $347 \mathrm{ppm}$ derived by Cartledge et al. (2004) within 800 parsecs. It is also interesting to compare with the range of solar photospheric $\mathrm{O} / \mathrm{H}$ values recently proposed by Grevesse et al. (2007). The value derived here from the TiII data, 480-500 ppm, is within their quoted range of 407-513 ppm, while the value derived from FeII, 300 ppm, is significantly below. These calculations suggest that the slopes derived from the linear fits using TiII are meaningful.

Is there something to learn about the local ISM history from the $\mathrm{D} / \mathrm{H}$ abundance pattern? The large-scale event that has given rise to the Gould belt of enhanced star formation is about $60 \mathrm{Myr}$ old (see e.g. Perrot \& Grenier 2003). Two main classes of scenarios have been proposed for the belt origin: the interaction between a giant external cloud and the disk on one hand (Comeron \& Tora 1994; Olano 2001) and a strong explosive event on the other (Olano 1982). Because the age of the belt is smaller than the mixing time of gases (a few hundreds Myr, see e.g. simulations by De Avillez \& Breitschwerdt 2004), in the former case of cloud collision the belt may have left imprints in the form of incomplete mixing between disk and "fresh" gas. In the latter case there must be dust evaporation at the shocked front regions of the belt but no gas mixing. A link between the Gould belt expansion and the $\mathrm{D} / \mathrm{H}$ distribution has been suggested because highly variable D/H regions shown by Linsky et al. (2006, Fig. 1) are located at distances (inferred from the $\mathrm{H}$ columns) that roughly correspond to the front of the expanding Gould belt of young stars and supernovae (Lallement 2007). Whatever the origin of the belt, the subsequent and observed expansion produces star formation and supernovae at the periphery of the belt, i.e. evaporation of dust in the shocked and heated regions of its periphery, which could explain the observed variability of $\mathrm{D} / \mathrm{H}$ at intermediated columns $\left(10^{19.5-20.5} \mathrm{~cm}^{-2}\right)$. The difference between the two scenarios for the belt origin is that in the cloud collision case some deuterium abundance variability due to gas mixing must add to abundance variation due to grain destruction, and there must be a larger amount of less astrated gas within the Gould belt as compared with outside the belt. There are no signs of astration variations that follow from the present correlative analysis, in agreement with the constancy of $\mathrm{O} / \mathrm{H}$ up to large distances (André et al. 2003). If D and metal abundance variability has some link with the belt, then the absence of signs of differential astration at columns lower than $10^{21} \mathrm{~cm}^{-2}$ seems to favor the explosive event scenario.

Independantly of a potential effect of the Gould belt on the $\mathrm{D} / \mathrm{H}$ distribution, our results provide a support for the ideas, originally suggested by Draine et al. (2004) and Linsky et al. (2006), that time-dependent deuterium depletion onto dust grains is responsible for the variability of the $\mathrm{D} / \mathrm{H}$ ratio, and for the low values of $\mathrm{D} / \mathrm{H}$ observed for many lines of sight beyond the Local Bubble. On the other hand, it remains to explain why very high $\mathrm{D} / \mathrm{H}$ ratios do not correspondingly present very high $\mathrm{D} / \mathrm{O}$ values.
This is important for the precise determination of the present galactic $\mathrm{D} / \mathrm{H}$ ratio.

Acknowledgements. We thank our referee Jeffrey Linsky for very carefully reading of the manuscript. His remarks and criticisms resulted in a strong improvement of the paper.

We thank Francis Dalaudier from Service d'Aéronomie for his useful recommendations on the fitting procedures and interesting suggestions for non symmetric error treatments, and Alain Lecavelier des Etangs from IAP for his support of the observing proposal and useful discussions.

\section{References}

André, M. K., Oliveira, C. M., Howk, J. C., et al. 2003, ApJ, 591, 1000 Asplund, M., Grevesse, N., \& Sauval, A. J. 2005, in ASP Conf Ser. 336, ed. Barnes, \& Bash, 25

Boggs, P. T., Byrd, R. H., Donaldson, J. R., \& Schnabel, R. B. 1989, Algorithm 676 - ODRPACK: Software for Weighted Orthogonal Distance Regression, ACM Trans. Mathematical Software, 15, 348

Cartledge, S. I. B., Lauroesch, J. T., Meyer, D. M., \& Sofia, U. J. 2004, ApJ, 613, 1037

Comeron, F., \& Torra, J. 1994, A\&A, 281, 35

de Avillez, M., \& Breitschwerdt, D. 2004, Ap\&SS, 289, 479

Dekker, H., D’Odorico, S., Kaufer, A., Delabre, B., \& Kotzlowski, H. 2000, Proc. SPIE, 4008, 534

Draine, B. T. 2004, in Origin and Evolution of the Elements, ed. A. McWilliams, \& M. Rauch (Cambridge: Cambridge Univ. Press), 320

Ellison, S. L., Prochaska, J. X., \& Lopez, S. 2007, MNRAS, 380, 1245

Field, G. B., \& Steigman, G. 1971, ApJ, 166, 59

Friedman, S. D., Hébrard, G., Tripp, T. M., Chayer, P., \& Sembach, K. R. 2006, ApJ, 638, 847

Geiss, J., Gloeckler, G., \& Charbonnel, C. 2002, ApJ, 578, 862

Grevesse, N., Asplund, M., \& Sauval, A. J. 2007, Space Sci. Rev., 130, 105

Hébrard, G., \& Moos, H. W. 2003, ApJ, 599, 297

Hébrard, G., Mallouris, C., Ferlet, R., et al. 1999, A\&A, 350, 643

Hébrard, G., Tripp, T. M., Chayer, P., et al. 2005, ApJ, 635, 1136

Hobbs, L. M. 1984, ApJS, 56, 315

Hoopes, C. G., Sembach, K. R., Hébrard, G., Moos, H. W., \& Knauth, D. C. 2003, ApJ, 586, 1094

Jenkins, E. B., Tripp, T. M., Woźniak, P. A., Sofia, U. J., \& Sonneborn, G. 1999, ApJ, 520, 182

Jura, M. 1982, in Advances in Ultraviolet Astronomy, ed. Y. Kondo, NASA CP238,54

Lallement, R. 2007, Space Sci. Rev., 130, 341

Lallement, R., Welsh, B. Y., Vergely, J. L., Crifo, F., \& Sfeir, D. 2003, A\&A, 411,447

Lemoine, M. 2002, ApJS, 140, 67

Linsky, J. L. 1998, Space Sci. Rev., 84, 285

Linsky, J. L. 2007, Space Sci. Rev., 130, 367

Linsky, J. L., Draine, B. T., Moos, H. W., et al. 2006, ApJ, 647, 1106

Millour, F., Petrov, R. G., Chesneau, O., et al. 2007, A\&A, 464, 107

Moos, H. W., Sembach, K. R., Vidal-Madjar, A., et al. 2002, ApJS, 140, 3

Morton, D. C. 1991, ApJS, 77, 119

Olano, C. A. 1982, A\&A, 112, 195

Olano, C. A. 2001, AJ, 121, 295

Oliveira, C. M., \& Hébrard, G. 2006, ApJ, 653, 345

Oliveira, C. M., Hébrard, G., Howk, J. C., et al. 2003, ApJ, 587, 235

Oliveira, C. M., Dupuis, J., Chayer, P., \& Moos, H. M. 2005, ApJ, 625, 232

Oliveira, C. M., Moos, H. W., Chayer, P., \& Kruk, J. W. 2006, ApJ, 642, 283

Perrot, C., \& Grenier, I. 2003, A\&A, 404, 519

Prochaska, J. X., Tripp, T. M., \& Howk, J. C. 2005, ApJ, 620, L39

Pickering, J. C., Thorne, A. P., \& Perez, R. 2001, ApJS, 132, 403

Rogerson, J. B., \& York, D. G. 1973, ApJ, 186, L95

Romano, D., Tosi, M., Chiappini, C., \& Matteucci, F. 2006, MNRAS, 369, 295

Sahnow, D. J., Moos, H. W., Ake, T. B., et al. 2000, ApJ, 538, L7

Sfeir, D. M., Lallement, R., Crifo, F., \& Welsh, B. Y. 1999, A\&A, 346, 785

Snowden, S. L., Egger, R., Finkbeiner, D. P., Freyberg, M. J., \& Plucinsky, P. P. 1998, ApJ, 493, 715

Sonneborn, G., Tripp, T. M., Ferlet, R., et al. 2000, ApJ, 545, 277

Stokes, G. M. 1978, ApJS, 36, 115

Wallerstein, G., \& Goldsmith, D. 1974, ApJ, 187, 237

Welsh, B. Y., Sasseen, T., Craig, N., Jelinsky, S., \& Albert, C. E. 1997, ApJS, 112,507

Welsh, B. Y., Sfeir, D. M., Sirk, M. M., \& Lallement, R. 1999, A\&A, 352, 308

Welty, D. E., Morton, D. C., \& Hobbs, L. M. 1996, ApJS, 106, 533 